\title{
DESCUBRIR LOS PAISAJES DE LAS AMÉRICAS. DISEÑAR, PLANIFICAR, CONSERVAR Y GESTIONAR. CARTA DEL PAISAJE DE LAS AMÉRICAS.
} Raquel Peńalosa, Saúl Alcántara, Carlos Jankilevich, lucia Veras, María Teresa Ocejo Cázares (Redactores) Universidad Autónoma Metropolitana, Azcapotzalco, México\&Federación Internacional

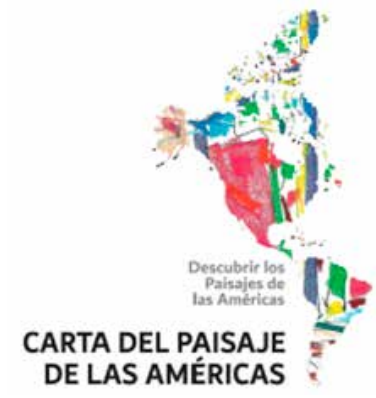

\section{PREFACIO}

En el marco del cuadragésimo séptimo Congreso Internacional de Arquitectura de Paisaje de la Federación Internacional de Arquitectos Paisajistas (IFLA), que tuviera lugar en Suzhou, en China, en el año 2010, los países miembros de esta organización se plantearon el compromiso de impulsar las cartas nacionales de paisaje de cada uno de ellos, como parte del proceso de elaboración de una Carta y eventualmente una Convención del Paisaje a nivel global.

La Carta, estaría respaldada por la Organización de las Naciones Unidas para la Educación, la Ciencia y la Cultura (UNESCO), que es un organismo especializado del Sistema de las Naciones Unidas (ONU), y dentro de sus contenidos, se destacaba el tema del derecho al paisaje como bien colectivo. Desde los inicios ha quedado claro que la Carta Global es de cierta complejidad, en virtud de las diferentes acepcio- nes de paisaje propias de la amplia diversidad geográfica, histórica, social, ambiental y cultural de quienes la definirían universalmente.

A partir del encuentro de Suzhou, las Cartas Nacionales de Paisaje se han incrementado en número, organizándose en algunos casos a nivel regional o continental, hasta abarcar los cinco bloques mundiales de IFLA.

En el caso de las Américas, el liderazgo pionero correspondió a Colombia, país que ha generado un movimiento transnacional denominado La Iniciativa Latinoamericana del Paisaje (LALI), que impulsó la redacción de la Carta Latinoamericana de Paisaje, cuya concepción al evolucionar, hacia el paisaje del Continente en su totalidad, llega a ser designada como la Carta del Paisaje de las Américas.

El propósito de la Carta es, entre otros, favorecer la toma de consciencia de la recupe- 
ración y puesta en valor del paisaje, así como construir instrumentos que favorezcan del desarrollo de un marco legal orientador, a partir de la realidad presente, considerando el pasado para construir el futuro.

De los treinta y cinco países que comprende el continente americano, diez y nueve son asociaciones nacionales de IFLA Américas.

Dieciséis de ellas, han formulado su Carta Nacional de Paisaje, la mayoría promueven la protección, la planificación y la gestión sostenible de los paisajes. Algunas, en forma explícita, se adhieren a los fundamentos de la Carta y Convenio Europeo del Paisaje. En su conjunto privilegian el reconocimiento de sus identidades y singularidades del carácter y valores a ser resguardados. Un importante número de ellas, subraya un rasgo definitorio de la Carta del Paisaje de las Américas: ser un instrumento de planificación y acción que garantiza el derecho a la felicidad como un bien colectivo patrimonial.

La Carta del Paisaje de las Américas está basada en la búsqueda de nuestras raíces y de la razón de ser de nuestra existencia, a partir del conocimiento de quiénes somos y qué poseemos como habitantes del mismo. Tal demanda es una de las razones fundamentales que ha motivado la elaboración de las Cartas Nacionales del Paisaje y es un rasgo distintivo de su marco conceptual.

La comprensión del paisaje americano se da a partir de una identidad particular; la "americanidad". Aquella del diálogo permanente entre la diversidad del territorio y la unidad constante en la cultura. Es con este espíritu que presentamos la Carta del Paisaje de las Américas.
PREÁMBULO: (RE) DESCUBRIENDO EL PAISAJE DE LAS AMÉRICAS

El Continente Americano ocupa el segundo lugar, en cuanto al tamaño, con respecto a los cinco continentes del planeta, con sus treinta y cinco países y dieciocho dependencias, es un territorio con una gran diversidad fisiográfica que nos desafía a mirarlo como una unidad compuesta por pluralidades y singularidades.

Para la elaboración de esta Carta, más que describir los paisajes, se ha adoptado la modalidad de individualizar, separar los estratos que lo componen, para después reestructurarlas con una mayor comprensión del paisaje de un continente único con una historia milenaria.

Cinco son los estratos definidos: naturaleza, cosmovisión, cultura, ética y "americanidad" como identidad. Tales estratos permiten tomar conciencia de que la atención primordial no es en los Paisajes de América, sino en el

\section{Paisaje de las Américas.}

El primer estrato se refiere a la na-

turaleza primigenia, la comprendida como patrimonio biológico sobre el cual se erigió el Continente Americano. Al derramarse de norte a sur, las Américas definen en su fisiografía, la conexión continental excepcional entre los dos polos terrestres, desde Alaska a la Patagonia, situándose entre los océanos Glaciar Ártico, Glaciar Antártico, Atlántico y Pacífico. Se desarrolla de norte a sur en grandes masas territoriales, en fragmentadas islas de distintas configuraciones, incluso en franjas delgadas de territorios que permiten la unión entre el Pacífico y el Atlántico.

Es un Continente plural que se articula por su heterogeneidad climática resultan- 
te de la confluencia de variación de latitudes, altitudes e influencias costeras, tierras altas de montañas y bajas en la línea del mar. Estos pisos ecológicos definen la identidad que se va construyendo del trabajo de adaptación impuesta por la naturaleza a los pueblos americanos, comprendiendo este soporte como un todo vivo.

Reconocer la superposición que culturalmente se va construyendo sobre la naturaleza prístina, constituye el segundo estrato del paisaje americano, aquello que se refiere al aspecto metafísico del paisaje, del dominio de la cosmovisión que incorpora la espiritualidad, la sacralidad, los sentimientos del alma y la estética filtrada por el arte, el misticismo de lo épico y de lo trágico, de lo simple y de lo sublime asociados al imaginario individual y colectivo, de los pueblos originarios y aún de los contemporáneos.

La diversidad del legado biológico de las Américas, ha exigido como condición de supervivencia las diferentes formas de adaptación y apropiación de la naturaleza como recurso, que crearon vínculos e interacciones con la "madre tierra", y con el árbol que tiene "alma propia", basados en creencias y valores espirituales trascendentales, con los cuales regular los astros y la naturaleza, con derivaciones directas en sus sistemas de subsistencia.

La cosmovisión es una forma mágica de ver la vida y entender la naturaleza, fruto de la relación que el ser humano estableció con la tierra y con sus dioses, por ejemplo en la Mesoamérica - Prehispánica, la cual da sentido a su existencia a través del paisaje. Este estrato del paisaje se refiere a la cosmogonía y posición del ser humano en el universo, así como del cosmos que lleva dentro de sí -cuerpo y mente-, lo cual se exterioriza en el territorio para formar el paisaje americano.

Tercer estrato del paisaje, la superposición en el espacio y el tiempo. La visión del paisaje, como un palimpsesto cultural del continente americano: (1) los pueblos originarios, (2) los colonizadores mezclados a los pueblos originarios de las tierras ya conquistadas, (3) el pueblo "americano" del día de hoy y sus distintos paisajes del norte, del centro y del sur de las Américas.

El primer palimpsesto apunta las influencias de la naturaleza que definieron una ocupación más nómada al norte y al sur, contrastando con la templada Mesoamérica, donde los relictos de paisaje quedan señalados por la arquitectura de los pueblos originarios, conocida como prehispánica.

El segundo palimpsesto, caracterizado por la mezcla entre indígenas y conquistadores, que han propiciado extraordinarios paisajes urbanos y rurales.

El tercer palimpsesto determina el paisaje contemporáneo, producto del proceso de industrialización que revela semejanzas entre el centro y el sur de América Ibérica y diferencias entre la América Ibérica y la América Anglosajona, desde el punto de vista económico, social y cultural. Es el palimpsesto de la contemporaneidad, urbanizado, en donde las ciudades son ocupadas por la mayoría de la población mundial, que impone condiciones especiales de planificación.

El cuarto estrato acusa un cambio de paradigma que se contrapone a la idea de que la naturaleza es inagotable y del desarrollo ilimitado, cuando el punto de inflexión está en la relación entre ética ambiental y estética, o sea, al considerar la naturaleza como ente estético y a su vez con un estatuto de ética. 
Si la modernidad nos ha alejado de la naturaleza para comprenderla como paisaje, la relación de interdependencia nunca se ha interrumpido y ha impuesto ese retorno a la natura, para alejarnos de las incertidumbres derivadas de la insensatez tecnológica, que repercute ambiental, económica y socialmente, lo cual crea abismos entre bienestar y pobreza. Pensar en la naturaleza como condición de existencia, nos aparta del conocimiento del paisaje únicamente como panorama, entendido al ser humano como contemplador, más bien el ser humano es parte de la naturaleza de la cual depende su existencia y espiritualidad. La ética ambiental se propone considerar al mundo natural como valor moral.

El quinto estrato relaciona a todos los demás con la tarea de comprender sus interrelaciones e interdependencias, en el sentido de nuestra "americanidad" como identidad. Las razones de este entendimiento se fundamentan en la recomposición de los cinco estratos superpuestos como valores, que en su conjunto nos definen una nueva mirada sobre qué es ser americano y cómo se define el paisaje.

Las Américas como un solo continente, entrelazado transversalmente en los cinco estratos que redescubren el paisaje americano, posibilita comprensión de la "americanidad" como condición de desarrollo con calidad, de la importancia que este concepto fundamenta la conservación, la planificación, el diseño y la gestión de nuestros paisajes pretéritos, actuales y del futuro, para responder a un objetivo principal: centrarse en la búsqueda, en la recuperación y consolidación de la felicidad como un derecho y como una conquista para todos los americanos.

\section{CONSIDERANDOS}

Los miembros de IFLA-Américas y signatarios distinguidos que firman el presente documento, consideran:

La necesidad de establecer una mirada especial para el paisaje de las Américas, respetando y valorizando la unidad de su diversidad y singularidades físico-geográficas, históricas, religiosas y socioculturales, a fin de fortalecer entre sus miembros, el sentido de americanidad que debe manifestarse en el diseño de nuevos paisajes, en la conservación del patrimonio cultural y natural, en la planificación que envuelve el pasado, el presente y el futuro de los paisajes y en la gestión que asegura la salvaguardia de las posturas que respetan el paisaje de las presentes y futuras generaciones;

La necesidad de reposicionamiento de la mirada hacia una noción de paisaje entre los que planean, diseñan, actúan y deciden el destino de los paisajes americanos, para incorporar el carácter simbiótico de la naturaleza y la cultura que envuelve y exige esa comprensión, en un proceso continuo de educación patrimonial sobre el paisaje, considerado como un bien de derecho universal.

Los paradigmas que mantienen la insostenibilidad como daños a la conservación de la biodiversidad de las Américas y consecuentemente del planeta, asociados a un desarrollo insostenible que explota los recursos naturales como inagotables, sin considerar el necesario equilibrio entre la preservación de la naturaleza, el abastecimiento de las necesidades sociales y la producción económica;

El cambio climático y el calentamiento glo$\underline{b a l}$, como una de las preocupaciones cruciales que repercuten en la conservación de la naturaleza y el paisaje, provocados por el aumento de 
la concentración de gases de efecto invernadero $\left(\mathrm{CO}_{2}\right.$, metano, óxido nitroso y clorofluorocarbonos), provenientes de actividades emitidas por las quemas en los campos y, principalmente, por las grandes ciudades donde los elementos patógenos son los protagonistas;

La necesidad de reconocer a las ciudades como paisajes urbanos que exigen tratamientos especiales, por requerir grandes flujos de energía, producir y acumular gran cantidad de basura, producir gases que contribuyen al calentamiento global y por ser, principalmente, el lugar de vivienda de la mayoría de la población del planeta, que concentra a la 'naturaleza' en forma de paisaje, en las plazas, parques, jardines, remanentes urbanos y espacios públicos;

Reconocer la fragilidad de los paisajes como elementos de cultura y como patrimonio no renovable, no sólo de ejemplares notables de la arquitectura, sino también de la arquitectura y paisaje vernáculos, que lleva formas de vida de comunidades que testimonian la diversidad de las culturas americanas;

Elpapel yla responsabilidad del Arquitecto del Paisaje en la preservación, conservación y la producción de nuevos paisajes, por ser el profesional que incorpora la relación entre naturaleza y cultura, en diferentes escalas de espacio, de tiempo y puede proponer proyectos que consideren la estética y la sustentabilidad de los lugares, alejándose del concepto de "tabla rasa" o de la "museificación" de los lugares, para mantener un compromiso respetuoso y creativo en el diálogo con los lugares, o sea, con los paisajes preexistentes.

\section{PRINCIPIOS}

Fundamentándose en las consideraciones señaladas, el presente documento establece como soporte a esta Carta, los siguientes Principios:
Incorporar todo lo vivo, como condición primera que precede a todos los demás Principios, por situarnos en la base vital y fisiológica de nuestra existencia como parte constituyente de la naturaleza, de cuya conciencia y respeto al todo vivo, depende la conservación y no la extinción de la vida en el planeta. La comprensión del todo vivo es un deber de todos y el todo vivo como paisaje, es un derecho de todos;

Recuperar la cosmovisión y la visión de la sacralidad, como una de las características intrínsecas que definen la formación del americano, fruto de la relación que los pueblos originarios establecieron con la tierra y con sus dioses, con el cosmos que da sentido a su existencia. La cosmovisión es una forma de ver la vida que continúa expresada en la contemporaneidad del paisaje americano, reverberándose en las tradiciones, costumbres y en el arte, donde la conciencia sensible no se apoya en conceptos, sino en las vivencias, en la herencia construida de la relación ancestral establecida entre los primeros americanos y las tierras de América.

Contemplar las singularidades del palimpsesto del territorio en escalas de espacio y de tiempo con el fin de reducir las desigualdades sociales y para mantener la identidad de las Américas, expresada en tres momentos distintos: (1) el palimpsesto de los pueblos originarios, más claramente presente en Mesoamérica - Prehispánica; (2) el palimpsesto resultante de la mezcla entre colonizados y colonizadores, que definió la arquitectura de innumerables ciudades y paisajes que se originaron por la explotación de minerales y de la producción agrícola; (3) el palimpsesto de la contemporaneidad, que se expresa, esencialmente, en las grandes ciudades, lugar de concentración po- 
blacional planetaria y de las grandes innovaciones tecnológicas, que vienen exponiendo, en el paisaje, no una relación con los lugares preexistentes, sino en otra escala, con un sistema global.

Retomar la ética comprendida en su relación con la estética, como condición íntimamente asociada a la ética ambiental cuando se propone rescatar el vínculo ancestral, entre el hombre y la naturaleza, en un entendimiento más allá de los parámetros antropocéntricos, cuando el paisaje gana además de sus cualidades estéticas con la objetividad y subjetividad de la belleza, el entendimiento del mundo natural como valor moral.

(Re) Descubrir las raíces de la americanidad como condición de futuro, es el principio que superpone a todos los otros en el redescubrimiento de las raíces que nos dan cimientos como americanos y nos permiten seguir proyectando el porvenir del paisaje, para consolidar nuestra identidad. La condición de futuro está asociada al respeto a la naturaleza, a la cultura, a los valores históricos y sociales, a las singularidades de la diversidad y al derecho al paisaje como un bien común y patrimonio colectivo, que también incorpora el derecho a la felicidad para todos.

\section{OBJETIVOS}

Fundamentándose en las consideraciones y principios establecidos, el presente documento establece como objetivos de la Carta del Paisaje de las Américas:

Ser un instrumento capaz de contribuir a la conquista de la felicidad como derecho pleno de todos los americanos.

Ser un instrumento de cohesión de un pensamiento americano sobre paisaje, que desde el punto de vista de su comprensión y de la definición de una política de protección, gestión y definición de nuevos paisajes, interconecte las tres Américas en un solo continente, respetándose y valorizando la diversidad y singularidades que les componen;

Establecer un marco legal que auxilie la aproximación entre la América anglosajona y la América Ibérica, para disminuir las diferencias económicas y sociales históricamente establecidas entre ricos y pobres, con el respecto de las singularidades físicas, históricas y culturales que distinguen cada una de estos territorios;

Asumir el papel legal de instrumento orientador para la conservación, la planificación, el diseño y la gestión del paisaje de las Américas, manteniendo coherencia con la legislación internacional que esté en consonancia con el desarrollo sostenible, con la valorización de la diversidad sociocultural y preservación de la calidad de vida;

Subsidiar las tomas de decisión en la planificación, en los planes de conservación, en los programas de gestión y en los diseños de proyectos de nuevos paisajes para que todos los gestos y acciones potencien los valores e caracteres presentes en los paisajes americanos, tangibles e intangibles, para reforzar sus singularidades como cualidades intrínsecas que definen nuestra identidad;

Estimular la participación del arquitecto del paisaje en las acciones gubernamentales de transformación del territorio, enfatizando su actuación en cargos de coordinación y de administración pública, por ser un profesional capaz de articular, de forma interdisciplinaria y en diferentes escalas, variables que involucran la preocupación socio-ambiental, cultural, espiritual, ecológica, estética, técnica y económica; 
Ser un instrumento orientador para los arquitectos del paisaje para que, conscientemente, incorporen los valores de las Américas, tangibles e intangibles -que incluya la vegetación y fauna nativas y endémicas de cada territorio-, en sus proyectos de paisajes futuros;

Ser un instrumento que pueda orientar las propuestas de intervención en los paisajes no solamente de arquitectos paisajistas, sino de todos los demás profesionales, de diversas áreas del conocimiento, así como las comunidades que trabajan en la gestión y conservación, que tienen el paisaje como objeto e interés de sus intervenciones;

Integrar el paisaje, con su debida importancia, en políticas públicas sectoriales (movilidad, infraestructura gris y verde, producción, explotación de recursos, energías renovables, salud, educación, turismo, y vivienda);

Desencadenar un proceso de educación paisajística y patrimonial para que la comprensión del paisaje -como un complejo sistema que relaciona la biodiversidad de la naturaleza y valores culturales-, sea incorporada a la gestión pública, respetando el derecho de todos a lugares saludables, dotados de los cuidados estéticos y ambientales, sea de paisajes de lo cotidiano o de valor patrimonial;

Estimular a las universidades e instituciones de educación superior de las Américas para incrementar los cursos de licenciatura y posgrado en las diversas escalas de la arquitectura del paisaje;

Promover e incorporar la participación social como condición necesaria para las decisiones públicas de planificación, conservación, gestión y diseño de los paisajes americanos, además de promover la participación de la po- blación en los proyectos y actividades de los planes de manejo del paisaje;

Resaltar la importancia de reconocer en un instrumento legal, la unidad de nuestra diversidad, tomando esas singularidades como valores que nos distinguen e identifican como continente americano, tanto para los paisajes de áreas naturales protegidas, como para los paisajes agrícolas, paisajes urbanos e integrar la protección de los bienes paisajísticos en la planificación urbana y regional, lo cual deberá cristalizarse en planes paisajísticos, del cual se derivan los demás instrumentos de ordenación territorial y urbana;

Reconocer las ciudades como paisajes urbanos que exigen cuidados y acciones especiales para que puedan cumplir su función social, acogiendo a la población con dignidad, calidad de vida y respetando los límites impuestos por la naturaleza.

Construir el inventario y el catálogo de los paisajes de las Américas, reconocidos como instrumentos fundamentales para la planificación, preservación y gestión.

Fomentar políticas públicas y participar en programas internacionales relativos al conocimiento de la cultura y la naturaleza, así como favorecer la cooperación regional en materia de la salvaguardia y construcción de paisajes.

Recuperación y puesta en valor del paisaje de las Américas como uno de los detonadores de la política cultural, de la recalificación urbana y recuperación ambiental de los países de la Región.

Ser un instrumento que contribuirá a la construcción del Convenio Global del Paisaje, respondiendo por el Continente Americano como la quinta pieza del "rompecabezas" planetario; 


\section{DEFINICIONES/GLOSARIO}

Fundamentándose en las consideraciones y principios establecidos, el presente documento establece como definiciones de la Carta del Paisaje de las Américas:

Paisaje: porción del territorio aprehendido por la experiencia sensible e inteligible de la percepción, individual y colectiva del ser humano que se revela como un unnicum y continuum de sistemas vivos, naturales y culturales, como una totalidad sintética e interdependiente, en el espacio y en el tiempo.

Conservación del paisaje: Consiste en definir un conjunto de acciones y estrategias con el propósito de mantener y transmitir al futuro, lo más posible, el mensaje cultural intrínseco del territorio, el trazado del pasado, de aquellos significados característicos de un paisaje, por su valor patrimonial como un documento - paisaje, considerando la naturaleza y la acción del ser humano como fruto de su acción sobre el sitio natural.

Planificación del paisaje: consiste en establecer las líneas de desarrollo sustentable del paisaje considerado éste como un bien primario, pero en relación con las exigencias y las necesidades de las sociedades, de lo preexistente y de los futuros sitios a desarrollar.

En este ámbito particular de desarrollo, tienen y tendrán un papel primordial las intervenciones de salvaguardia, preservación, defensa, recalificación del paisaje y la recuperación o rehabilitación de áreas deterioradas, tanto a nivel regional como en los ecosistemas urbanos, incluyendo paisajes únicos o característicos, en la búsqueda y creación de nuevos valores estéticos paisajísticos. Los fundamentos teóricos de esta escala de acción están basados en verdaderos estudios ecológicos con aportaciones de diversas disciplinas.

Diseño de Paisaje: es el arte de prever, en carácter prospectivo, la arquitectura de los espacios abiertos para un uso específico, con orden y calidad, tomando en cuenta las competencias ambientales y del contexto cultural propio de cada tejido urbano y/o entorno natural, con el propósito de mejorar, restaurar y/o crear nuevos paisajes, así como privilegiar el manejo de la vegetación nativa y endémica de cada territorio.

Gestión de paisaje: es el conjunto de acciones, estrategias y métodos definidos con el propósito de asegurar la conservación de un paisaje, así como orientar sus transformaciones, tomando en consideración las dinámicas resultantes de los procesos sociales, económicos y medioambientales, en una perspectiva de desarrollo sostenible, que debe incluir la efectiva participación social.

Política de Paisaje: de la competencia de las autoridades públicas, es la definición de un conjunto de principios, estrategias, directrices y acciones, generales y específicas, que orientan las medidas institucionales y no institucionales, sobre acciones que puedan interferir y modificar el paisaje, con vistas a su protección, gestión y ordenación.

\section{DECLARATORIA}

Nosotros, los signatarios de la Carta del Paisaje de las Américas nos comprometemos a actuar, tanto en el ámbito de nuestras actividades profesionales como en la vida cotidiana, conforme los considerandos aquí resaltados y respetando los valores y contenidos declarados, hacia la institucionalización de nuestros principios y conquista de los objetivos establecidos. Así 
mismo, manifestamos nuestra adhesión a todas las Cartas del Paisaje vigentes en las Américas, a los tratados y acuerdos vinculantes que hagan posible la puesta en práctica de las mismas, así como a la legislación internacional que esté en consonancia con el desarrollo sostenible, con la valorización de la diversidad sociocultural y preservación de la calidad de vida como un bien y derecho colectivo. Expresamos nuestra comprensión y responsabilidad de responder por las Américas y contribuir para la posible construcción y firma del Convenio Global del Paisaje.

Ciudad de México, 28 de Septiembre de 2018 Seminario de Cultura Mexicana

\section{COMITÉ REDACTOR}

- Saúl Alcántara Onofre, Universidad Autónoma Metropolitana, Azcapotzalco, México.

- María Teresa Ocejo Cázeres, Universidad Autónoma Metropolitana, Azcapotzalco, México.

- Raquel Peñalosa, Federación Internacional de Arquitectos Paisajistas.

- Carlos Jankilevich, Federación Internacional de Arquitectos Paisajistas.

- Lucia Veras Cavalcanti, Laboratorio de Paisaje, Universidad Federal de Pernambuco, Brasil.

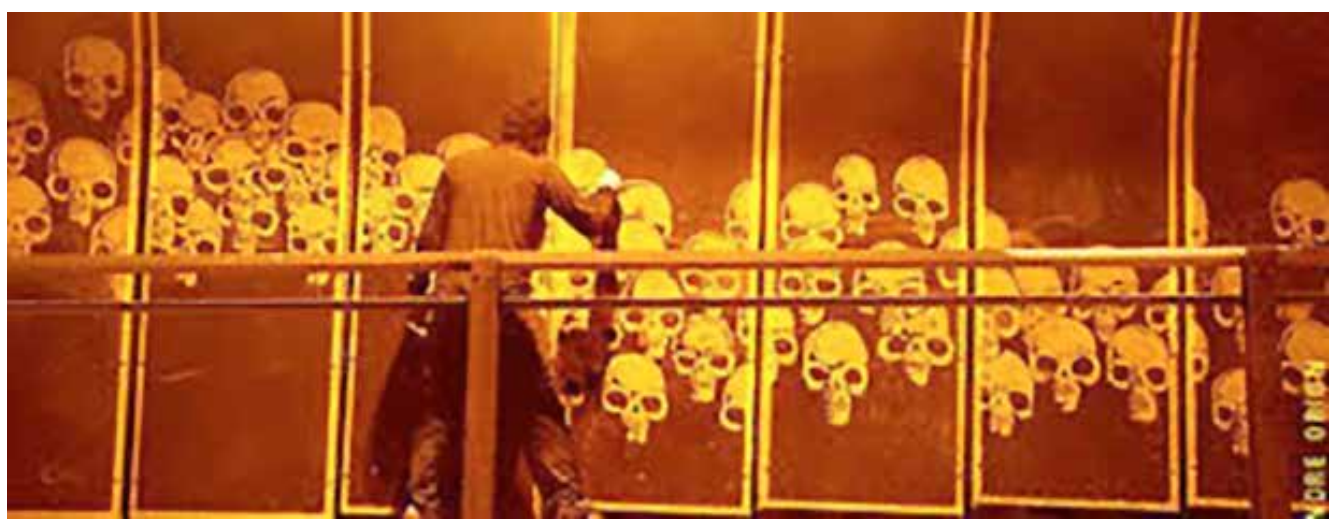

Ossário. 2006. 360 Metros de extensão. Túnel max feffer. São paulo. Brasil 